\title{
A COMBINED FINITE ELEMENT AND MACHINE LEARNING APPROACH FOR THE PREDICTION OF SPECIFIC CUTTING FORCES AND MAXIMUM TOOL TEMPERATURES IN MACHINING*
}

\author{
SAI MANISH REDDY MEKARTHY ${ }^{\dagger}$, MARYAM HASHEMITAHERI ${ }^{\dagger}$, AND HARISH CHERUKURI ${ }^{\dagger}$
}

\begin{abstract}
In machining, specific cutting forces and temperature fields are of primary interest. These quantities depend on many machining parameters, such as the cutting speed, rake angle, tool-tip radius, and uncut chip thickness. The finite element method (FEM) is commonly used to study the effect of these parameters on the forces and temperatures. However, the simulations are computationally intensive and thus, it is impractical to conduct a simulation-based parametric study for a wide range of parameters. The purpose of this work is to present, as a proof-of-concept, a hybrid methodology that combines the finite element method (FE method) and machine learning (ML) to predict specific cutting forces and maximum tool temperatures for a given set of machining conditions. The finite element method was used to generate the training and test data consisting of machining parameter values and the corresponding specific cutting forces and maximum tool temperatures. The data was then used to build a predictive model based on artificial neural networks. The FE models consist of an orthogonal plane-strain machining model with the workpiece being made of the Aluminum alloy Al 2024-T351. The finite element package Abaqus/Explicit was used for the simulations. Specific cutting forces and maximum tool temperatures were calculated for several different combinations of uncut chip thickness, cutting speed and the rake angle. For the machine learning-based predictive models, artificial neural networks were selected. The neural network modeling was performed using Python with Adam as the training algorithm. Both shallow neural networks (SNN) and deep neural networks (DNN) were built and tested with various activation functions (ReLU, ELU, tanh, sigmoid, linear) to predict specific cutting forces and maximum tool temperatures. The optimal neural network architecture along with the activation function that produced the least error in prediction was identified. By comparing the neural network predictions with the experimental data available in the literature, the neural network model is shown to be capable of accurately predicting specific cutting forces and temperatures.
\end{abstract}

Key words. finite element modeling, machining, machine learning, artificial neural networks, activation function, shallow and deep networks, Adam, specific cutting forces, maximum tool temperature

AMS subject classifications. 74S05

1. Introduction. Orthogonal machining, shown in Figure 1.1, is a metal cutting process in which the cutting edge of the tool is perpendicular to the workpiece. The cutting forces

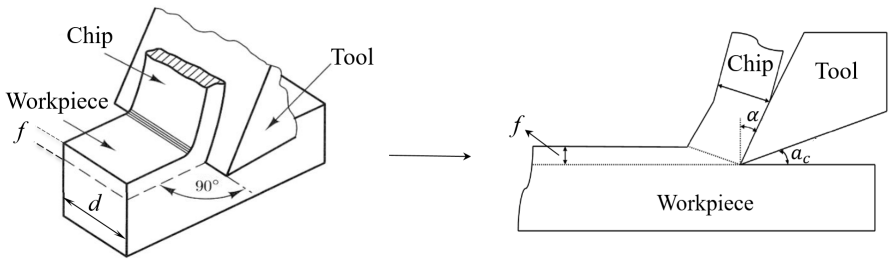

FIG. 1.1. Orthogonal machining [24].

and maximum tool temperatures are of practical interest. Once the cutting force is known, the specific cutting force, $K_{s}$, defined as the cutting force required to remove unit area of work material and mainly important for estimating the power and torque requirements during

*Received September 30, 2020. Accepted March 30, 2021. Published online on December 20, 2021. Recommended by Peter Benner.

$\dagger$ Department of Mechanical Engineering and Engineering Science, University of North Carolina at Charlotte, NC, 28223, USA (Harish. Cherukuri@uncc.edu). 
machining, is calculated from

$$
K_{s}=\frac{F_{c}}{f d},
$$

where $F_{c}$ is cutting force, $f$ is chip thickness, and $d$ is chip width.

Orthogonal machining is often modeled as a two-dimensional plane-strain problem. The FE models involve proper selection of a reliable constitutive model for material behavior, criterion for chip separation (damage modeling), and an appropriate contact formulation for modeling tool-chip interaction. The simulations, even when they are two-dimensional, are computationally intensive. Consequently, a comprehensive finite element study of the various parameters' effect on the cutting forces and temperatures is often impractical.

Predictive models based on machine learning offer an alternative approach. In recent years, a few studies have been reported in the literature involving the use of artificial neural networks (ANNs) for machining applications. ANNs are a data processing and modeling technique that arose in pursuit of mathematical modeling of the learning process based on the human brain. ANNs are effective as computational processors for various classification, regression, data compression, forecasting, and combination problem solving tasks [25]. Ovali et al. [26] conducted a study on predicting cutting forces in austempered grey iron using ANNs and concluded that they have more ability than regression analysis to solve problems having non-linear relationships. Kara et al. [16] also performed modeling of cutting forces during the orthogonal machining of AISI 316L stainless steel with cutting speed, feed rate, and coating type as the input parameters using both multiple regression and ANNs and concluded that results obtained from ANNs are predictive. Asokan et al. Al-Ahmari [3] and [5] also compared regression analysis with ANNs and concluded that ANNs are better in terms of performance.

Abdullah et al. [38] and Tasdemir in [35, 36] determined the best neural network architecture by monitoring statistical results obtained by computing the mean squared error (MSE) and the coefficient of determination $R^{2}$. The model with the least MSE and highest $R^{2}$ was selected to be the most suitable network architecture. A similar approach is used in this work.

Regarding the activation functions used in ANN modeling, Pontes et al. [30] stated that, eleven publications had used hyperbolic tangent activation functions and seven publications had used sigmoid activation function. Correa et al. [10] highlighted that there are no standard algorithms for choosing the network parameters; number of hidden layers, number of nodes in the hidden layers, and the activation functions. Haykin [11] in his work stated that hyperbolic tangent activation leads to faster convergence in training due to its symmetrical shape. He also added that there are no standard methods to determine the number of hidden layers and neurons. In this work, we consider several different activation functions along with deep and shallow neural networks to identify an optimal neural network architecture.

2. Problem statement. In this work, a finite-element (FE) model of orthogonal machining is developed first. This is followed by a validation of the model using data published in the open literature. The chip formation was simulated by using a recent fracture-based methodology introduced by Patel and Cherukuri [28]. Simulations are performed for various combinations of cutting speeds $V_{c}$, rake angles $\alpha$, and uncut chip thickness $f$. The data generated (i.e., maximum temperature and cutting forces) will be used to develop ANN-based predictive models. As both specific cutting forces and maximum tool temperatures are continuous values, ANNs are used for regression. Several neural network architectures, within shallow and deep networks, will be built along with the implementation of five different activation functions. The neural network architecture and the activation function that produces the least error in prediction is identified. In addition, sensitivity analysis is performed on the selected 


\section{ETNA}

Kent State University and

Johann Radon Institute (RICAM)

neural network to study the effect of input parameters on the output. Figure 2.1 shows the work flow.

\begin{tabular}{|c|c|c|c|c|}
\hline $\begin{array}{c}\text { Finite element } \\
\text { modeling }\end{array}$ & $\begin{array}{c}\text { Simulations and } \\
\text { data extraction }\end{array}$ & $\begin{array}{l}\text { ANN modeling } \\
\text { and analysis }\end{array}$ & $\begin{array}{l}\text { Identify suitable network } \\
\text { and activation function }\end{array}$ & $\begin{array}{c}\text { Sensitivity } \\
\text { analysis }\end{array}$ \\
\hline
\end{tabular}

FIG. 2.1. Work flow.

3. Finite element modeling. Here, we discuss the formulation, set up, and also material, contact, and damage modeling in our finite element simulations. The orthogonal machining process is simulated by solving a fully coupled thermal-structural and dynamic problem using Abaqus/Explicit. The workpiece is taken to be made of an aluminum alloy (Al 2024-T351) with tungsten carbide (WC) as the cutting tool.

3.1. Finite element model setup. The material properties for both the workpiece and the cutting tool are shown in Table 3.1. For model verification purposes, the geometry and the material properties are the same as those taken by [22]. A schematic of the computational model is shown in Figure 3.1. The workpiece and cutting tool are meshed using plane-strain, quadrilateral elements (CPE4RT) and triangular elements (CPE3RT) with reduced integration. The total number of elements used is 22447. The nodes on the bottom and left boundaries of the workpiece are fully constrained whereas the tool is given only horizontal motion (with cutting speed $V_{c}$ ) in the negative- $x$ direction. The clearance angle and the tool nose radius are $7^{\circ}$ and $20 \mu \mathrm{m}$, respectively.

TABLE 3.1

Material properties of workpiece and tool [22].

\begin{tabular}{lcr} 
Physical property & $\begin{array}{c}\text { Workpiece } \\
(\mathrm{Al} 2024-\mathrm{T} 351)\end{array}$ & $\begin{array}{r}\text { Tool } \\
\text { (WC) }\end{array}$ \\
\hline Density, $\rho\left(\mathrm{kg} / \mathrm{m}^{3}\right)$ & 2700 & 11900 \\
Young's Modulus, $E(\mathrm{GPa})$ & 73 & 534 \\
Poisson's ratio, $\nu$ & 0.33 & 0.22 \\
Specific heat, $(\mathrm{J} / \mathrm{kg} / \mathrm{K})$ & $C_{p}=0.557 T+877.6$ & 400 \\
Thermal expansion coeff., $\alpha_{d}\left(\mathrm{~K}^{-1}\right)$ & $\alpha=\left(8.9 e^{-3} T+22.6\right) e^{-6}$ & $\mathrm{NA}$ \\
Thermal conductivity, $(\mathrm{W} /(\mathrm{m} \cdot \mathrm{K}))$ & for: $25 \leq T<300$ & \\
& $\lambda=0.247 T+114.4$ & 50 \\
& for: $300 \leq T \leq T_{\text {melt }}$ & \\
& $\lambda=0.125 T+226$ & 50
\end{tabular}

3.2. Material modeling. The most widely used constitutive model in machining is the one proposed by Johnson-Cook [14]. The model has been shown by [2, 13, 27] to produce better results than other constitutive models. For this reason, in this work, the Johnson-Cook (JC) constitutive model is used for the thermomechanical response of the workpiece. The model is formulated empirically and it is based on von Mises plasticity, where von Mises yield surface (J2 plasticity theory) is associated with the flow rule. The JC constitutive equation assumes isotropic hardening and is capable of modeling thermo-visco-plastic problems over a 


\section{ETNA}

Kent State University and

Johann Radon Institute (RICAM)

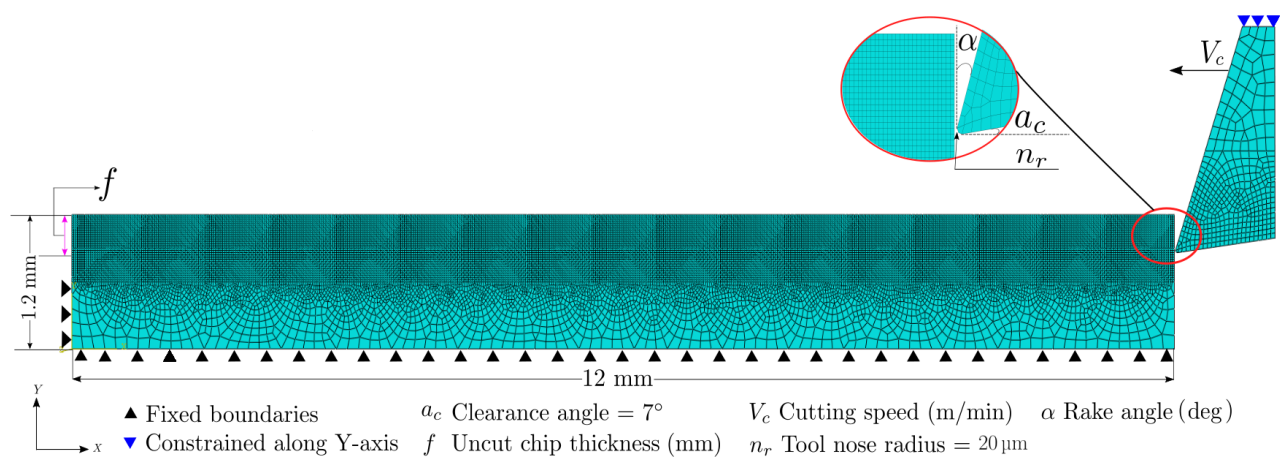

FIG. 3.1. Finite element model setup.

strain rate range of $10^{2}$ to $10^{5} \mathrm{~s}^{-1}$. The equation is given by

$$
\sigma(\bar{\epsilon}, \dot{\bar{\epsilon}}, T)=\left(A+B \bar{\epsilon}^{n}\right)\left[1+C \ln \left(\frac{\dot{\bar{\epsilon}}}{\dot{\epsilon}_{0}}\right)\right]\left[1-\bar{T}^{m}\right] .
$$

The flow stress is represented as a function of strain $\bar{\epsilon}$, strain rate $\dot{\bar{\epsilon}}$, and the non-dimensional temperature $\bar{T}$. The first term in the equation accounts for isotropic hardening whereas the second and third terms account for strain rate hardening and thermal softening respectively. The material parameters $A, B, n, C$, and $m$ for the JC model are given in Table 3.2 and are the same as used by $[22,37]$. Here, $\bar{T}$ in (3.1) is given by

$$
\bar{T}=\left\{\begin{array}{lc}
0, & T<T_{\text {trans }}, \\
\frac{T-T_{\text {trans }}}{T_{\text {melt }}-T_{\text {trans }}}, & T_{\text {trans }}<T<T_{\text {melt }}, \\
1, & T>T_{\text {melt }}
\end{array}\right.
$$

where $T_{\text {melt }}$ is the melting temperature and $T_{\text {trans }}$ is the transition temperature of the workpiece material.

TABLE 3.2

Johnson-Cook model parameters for Al 2024-T351.

\begin{tabular}{ccccccc}
$\begin{array}{c}A \\
(\mathrm{MPa})\end{array}$ & $\begin{array}{c}B \\
(\mathrm{MPa})\end{array}$ & $n$ & $C$ & $m$ & $\begin{array}{c}T_{\text {trans }} \\
(\mathrm{K})\end{array}$ & $\begin{array}{c}T_{\text {melt }} \\
(\mathrm{K})\end{array}$ \\
\hline 352 & 440 & 0.42 & 0.0083 & 1 & 25 & 520
\end{tabular}

3.3. Contact modeling. Contact modeling in the secondary deformation zone, at the interface of the chip and the rake face of the tool is critical for studying the thermal events at the chip-tool interface. importance. From experimental results, it has been found and verified that two contact regions may be distinguished in dry machining: the sticking region, and the slipping region [24]. Zorev proposed a friction model in [42], where he showed that the normal stress $\left(\sigma_{n}\right)$ in the secondary deformation zone is maximum at the tool tip and reduces to zero at a point where the chip loses contact from the rake face. Although Zorev's model is widely used to model friction at the tool-chip interface, it has some severe drawbacks. For example, in the slip zone $l_{\text {slip }}$, the coefficient of friction $\mu$ is assumed to be constant and independent of $\sigma_{n}[39]$. 
In the present work, to overcome the drawbacks associated with Zorev's model, the stress-based friction model proposed by Yang and Liu [40] consisting of both stick and slip regions was used. For further details on this model, the reader is referred to the work by Patel et al. [29]. The tool-chip interaction was defined using the penalty stiffness contact formulation where the tool was considered as master surface and the chip was considered as slave surface. In addition, the self-contact of the chip was also defined using penalty contact formulation.

3.4. Damage modeling. Chip formation takes place as a result of damage and fracture in a material due to the action of the cutting tool. Finite element simulations require a criterion to simulate chip separation from the bulk when the tool moves and interacts with the workpiece. The chip separation criterion should closely reflect the physics and mechanics of chip formation to achieve reliable results. In this work, the Johnson-Cook damage model [15] is used to model machining as a process resulting from damage and fracture in a material. According to this model the overall damage in a material occurs in two steps [1]: damage initiation and damage evolution.

Damage initiates in a material when the damage parameter $\omega$ defined as:

$$
\omega=\sum \frac{\Delta \bar{\epsilon}}{\bar{\epsilon}_{d}},
$$

equals or exceeds one. The numerator $\Delta \bar{\epsilon}$ is the increment in equivalent plastic strain, whereas the denominator $\bar{\epsilon}_{d}$ is equivalent plastic strain at the onset of damage initiation and is given by

$$
\bar{\epsilon}_{d}=\left[D_{1}+D_{2} \exp \left(D_{3} \frac{p}{\bar{\sigma}}\right)\right]\left[1+D_{4} \ln \left(\frac{\dot{\bar{\epsilon}}}{\dot{\epsilon_{0}}}\right)\right]\left[1+D_{5} \bar{T}\right] .
$$

The parameters $D_{1}$ to $D_{5}$ are shown in the Table 3.3. The parameter $D_{5}$ is zero which indicates that temperature does not have any effect on the damage initiation of aluminum [22].

TABLE 3.3

Johnson-Cook damage model parameters for Al 2024-T351.

\begin{tabular}{ccccc}
$D_{1}$ & $D_{2}$ & $D_{3}$ & $D_{4}$ & $D_{5}$ \\
\hline 0.13 & 0.13 & 1.5 & 0.011 & 0
\end{tabular}

The damage evolution is modeled using the damage variable $D$. It has a value of zero at the onset of damage and equal to unity when the stiffness of the element is completely degraded. Two commonly used laws for its evolution are the linear evolution and exponential evolution. According to linear evolution, the overall damage variable $D$ is defined as:

$$
D=\frac{\dot{\bar{u}} \bar{\sigma}_{y}}{2 G_{f}}
$$

where $\dot{\bar{u}}$ is the rate of equivalent plastic displacement, $G_{f}$ the critical energy release rate, and $\bar{\sigma}_{y}$ the yield stress after the onset of damage. When $D=1$ in an element, the element is considered to be completely degraded and removed from the model.

According to exponential evolution, the overall damage variable $D$ is defined as:

$$
D=1-\exp \left[-\int_{0}^{\bar{u}} \frac{\bar{\sigma}_{y} d \bar{u}}{G_{f}}\right] .
$$


Since $D$ approaches one when $\bar{u}$ approaches infinity, in Abaqus, $D$ is taken to be one when the total dissipated energy for each element approaches $0.99 G_{f}$.

In this work, exponential evolution is defined across the area of uncut chip thickness $(f)$ whereas linear evolution is defined for the remaining area; see Figure 3.2. This approach and the values of $G_{f}$ (critical energy release rate) are adopted from Patel and Cherukuri in [28].
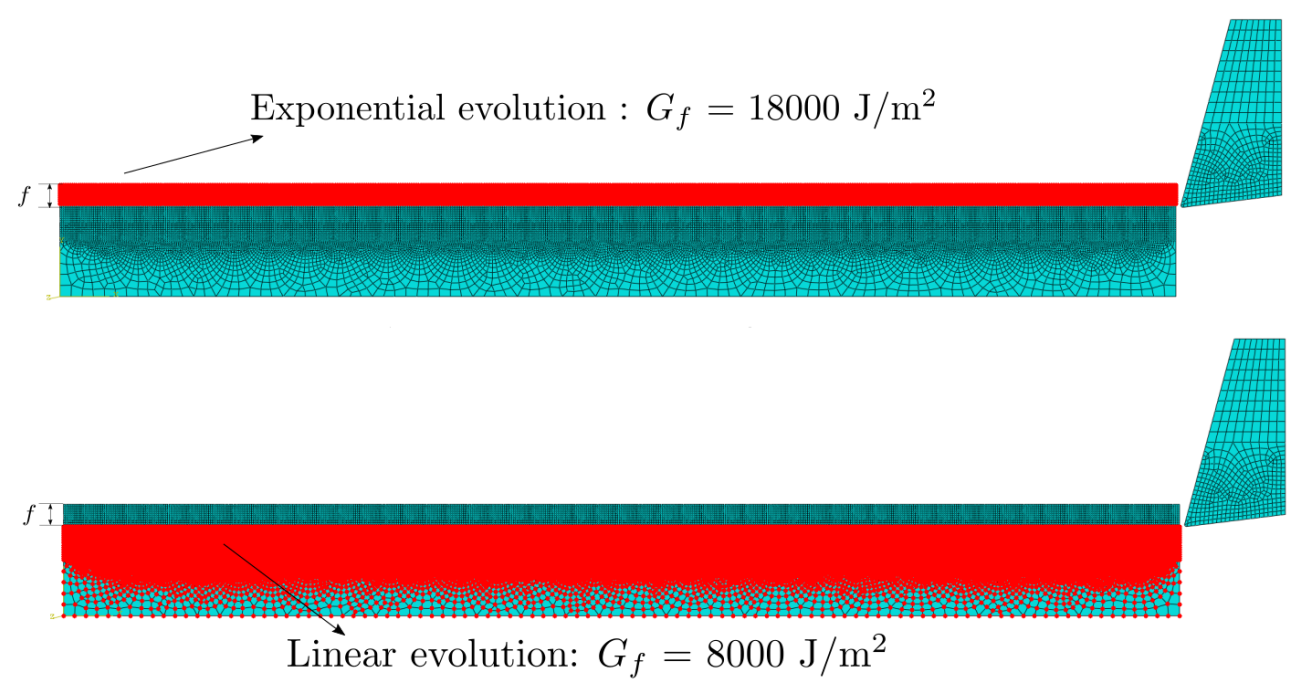

FIG. 3.2. Exponential and linear evolution.

4. Finite element model validation. In this section, the specific cutting forces and chip morphology obtained from the finite element analysis (FEA) simulations are compared with the experimental results available in the literature for similar cutting parameters.

The average specific cutting forces obtained from FEA simulations, for rake angle $17.5^{\circ}$ and uncut chip thicknesses of $0.3 \mathrm{~mm}$ and $0.4 \mathrm{~mm}$, during the cutting speeds of $200 \mathrm{~m} / \mathrm{min}$, $400 \mathrm{~m} / \mathrm{min}$, and $800 \mathrm{~m} / \mathrm{min}$ are compared with the available experimental results; Asad et al. [4]. Figures $4.1 \mathrm{a}$ and $4.1 \mathrm{~b}$ show the specific cutting forces and the corresponding difference respectively.

The difference for the results obtained for uncut chip thickness $0.4 \mathrm{~mm}$ is in the range $16 \%$ to $19 \%$, whereas for uncut chip thickness $0.3 \mathrm{~mm}$ the difference ranges from $15 \%$ to $17 \%$. Moreover, the specific cutting forces remain constant with the increase in cutting speed for the experimental data. Similar trend is observed for the results obtained from finite element simulations; see Figure 4.1a.

Chip morphology is a significant parameter to understand the material behavior in machining. It can be used as a primary parameter in optimizing the metal cutting process since it reflects the true measure of plastic deformation $[6,20]$. The obtained chip morphology is directly related to the cutting parameters chosen. A high rake angle or a large uncut chip thickness will result in serrations. Serrations occur due to the instability that arises due to interactions between strain hardening and thermal softening. Figure 4.2 shows the chip from our simulations for the rake angle $17.5^{\circ}$ and uncut chip thickness of $0.4 \mathrm{~mm}$ with a cutting speed of 800 $\mathrm{m} / \mathrm{min}$. This matches closely with the chip obtained in experiments by Mabrouki et al. [22].

5. FEA simulations and data extraction. The data for building ANN models is generated using the FE model described in Section 3 by varying cutting parameters: the rake angle, 


\section{ETNA}

Kent State University and

Johann Radon Institute (RICAM)

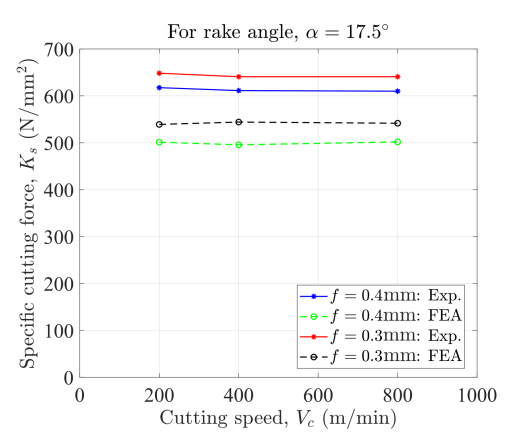

(a)

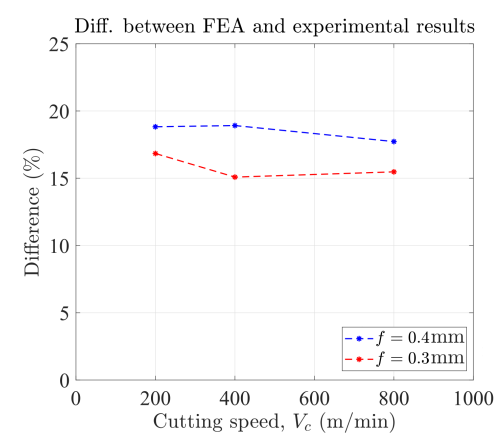

(b)

FIG. 4.1. Comparison of the specific cutting forces from FEA simulations with the experimental results (4.1a) and the corresponding difference (4.1b).

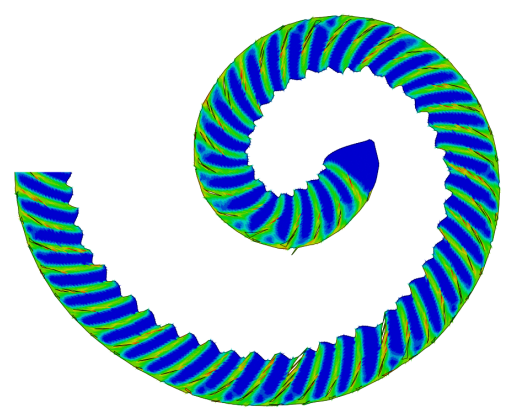

FIG. 4.2. Chip shape predicted by the finite element simulations the rake angle $17.5^{\circ}$ and uncut chip thickness of $0.4 \mathrm{~mm}$ with a cutting speed of $800 \mathrm{~m} / \mathrm{min}$.

uncut chip thickness, and cutting speeds. Simulations are performed for seven rake angles, four uncut chip thickness values and seven cutting speeds, see Table 5.1, resulting in a total of $196(7 \times 4 \times 7)$ simulations. The tool nose radius and clearance angle are kept constant for all the simulations. The total run time for all the simulations is approximately 2350 hours with each of the FE simulations taking 12 hours on average on Linux-based workstations with Intel-i7 CPU (3.6 GHz clock rate) and a minimum of $32 \mathrm{~GB}$ of RAM. The required output parameters (specific cutting force and maximum tool temperature) are obtained for all 196 simulations using a Python script.

6. Artificial neural networks. An artificial neural network structure consists of three main parts: the input, output, and hidden layers as shown in the Figure 6.1. The first (left) layer is the input layer and the last (right) layer is the output layer. The layer in between is hidden layer. Each of these layers has components called neurons; the ones shown as a circle in Figure 6.1. In a feed-forward neural network, also known as multilayer perceptron (MLP), the neurons in one layer are connected to the neurons in the next layer and the information flows forward from the input to the output through the hidden layers. The connections between the neurons are called synapses. Connections only exist between the neurons of two adjacent layers but not in the same layer.

A deep neural network (DNN) has more than one hidden layer (see Figure 7.2) whereas a shallow neural network (SNN) has only one hidden layer; see Figure 7.1. While deep neural 
TABLE 5.1

Parameters for simulations.

\begin{tabular}{ccc}
$\begin{array}{c}\text { Rake angle } \\
(\mathrm{deg})\end{array}$ & $\begin{array}{c}\text { Uncut chip thickness } \\
(\mathrm{mm})\end{array}$ & $\begin{array}{c}\text { Cutting speed } \\
(\mathrm{m} / \mathrm{min})\end{array}$ \\
\hline-3 & 0.1 & 100 \\
0 & 0.2 & 200 \\
5 & 0.3 & 400 \\
8 & 0.4 & 600 \\
15 & & 800 \\
17.5 & & 1000 \\
20 & & 1200
\end{tabular}

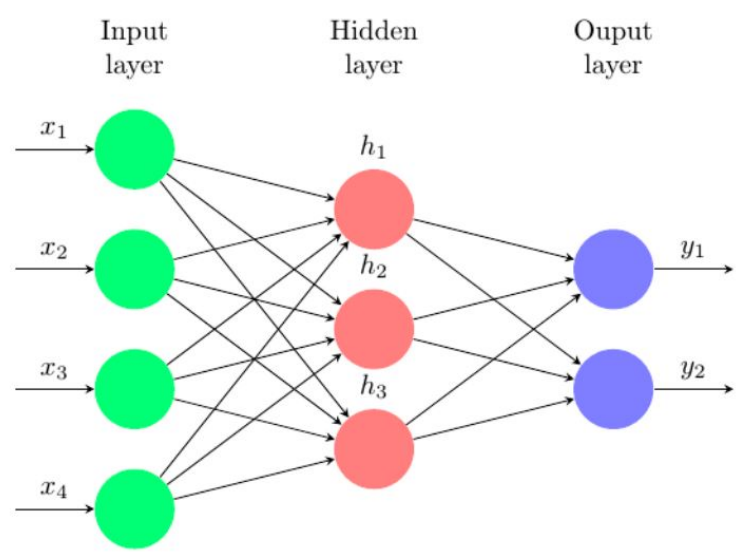

FIG. 6.1. An example of an artificial neural network (ANN).

networks usually outperform shallow networks on large datasets, for small datasets, shallow networks may perform just as well or even outperform deep networks in some cases [17, 21,33]. Neurons consist of a set of input values $x_{i}, i=1, \ldots, n$, a set of weights $w_{i}, i=1, \ldots, n$, and an activation function, $f$; see Figure 6.3. A linear transformation consisting of the weighted sum of all the inputs, $\sum w_{i} x_{i}$, and a bias $b$ is calculated as:

$$
z=b+\sum_{i=1}^{n} w_{i} x_{i}
$$

for each neuron [9]. The output $h$ is calculated from this neuron through the (usually) nonlinear activation function $f(z)$. Each neuron in a given layer has the same activation function and for each neuron $i$ in that layer, the output is calculated as $h_{i}=f\left(z_{i}\right)$, where $z_{i}$ is calculated using (6.1). The outputs serve as the inputs for each of the neurons in the next layer, which can use a different or the same activation function. The activation function transforms the received value into a real output through an algorithm. This process is continued until the output layer is reached where the neurons compute the output variables $y_{i}=1, \ldots, k$ with $k$ as the number of the outputs.

The activation functions are generally non-linear. Using non-linear activation functions allows ANN to be applied for complex problems. Few activation functions that are available in the software packages are sigmoid (logistic function), hyperbolic tangent sigmoid, softmax, ELU, ReLU, leaky ReLU, linear, etc. Some of the popular activation functions are illustrated 
in Figure 6.2. There are no standard methods available in the literature for the number of hidden layers, neurons, and the activation function. Hence, researchers follow a trial and error approach.
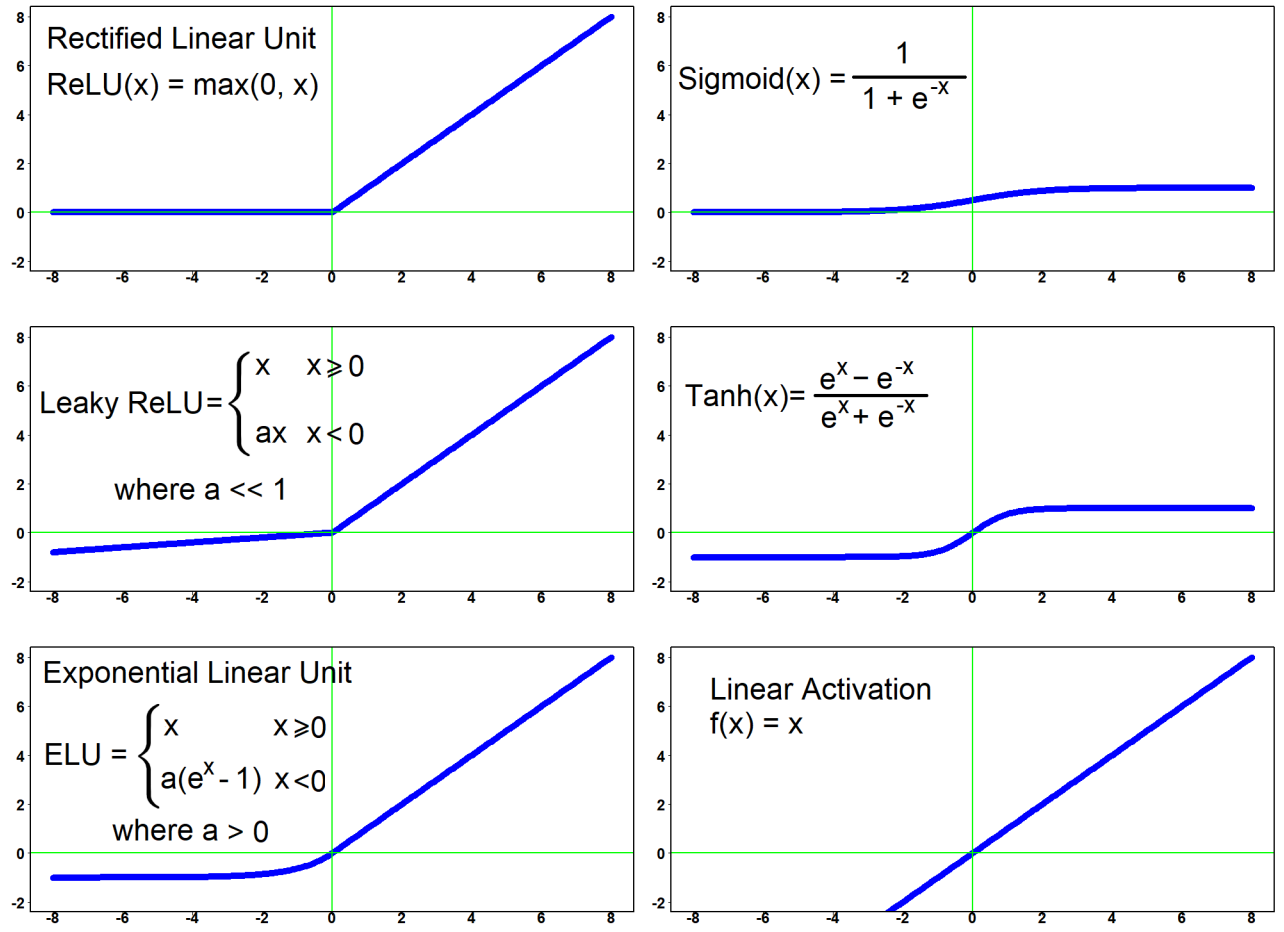

FIG. 6.2. Some of the popular activation functions. The activation functions are usually enable the neural networks to consider non-linearity in the data.

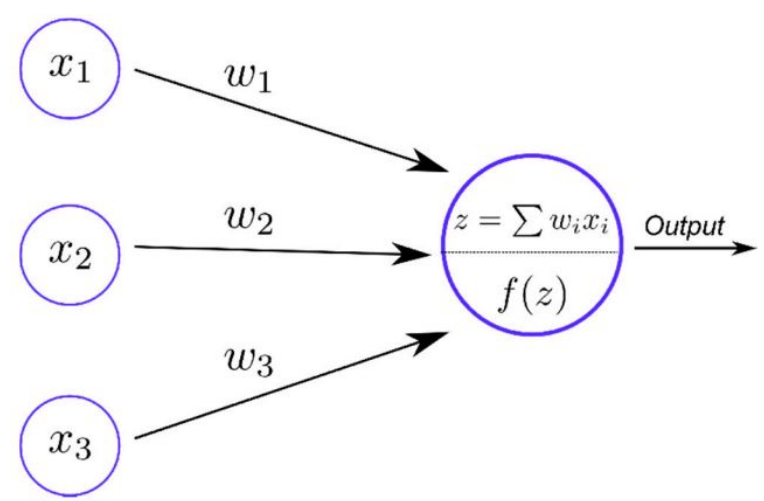

FIG. 6.3. A single neuron consists of the inputs $x_{i}$, the weights $w_{i}$, and the activation function $f(z)$, which produces the scalar value $h$. Note that $z$ is defined with the bias $b$ absorbed into the summation by setting $x_{0}=1$ and $w_{0}=b[8]$. 
In supervised learning, the training data (input data and the corresponding output data) is used to train the model. The training starts with an initial assumption on the weights $w_{i}$. The input data is processed by the ANN and output is predicted. The error between the predicted outputs and known outputs is calculated using a cost (or loss) function which can be the sum of the squares of the errors between predicted and observed outputs, for example. Since the predicted values depend on the weights and biases, it is clear that the loss function $E$ is also a function of the weights and biases for a given set of training data, i.e., $E=E\left(w_{i}, b\right)$. By absorbing the bias $b$ into the weights as an additional parameter, $E$ can be assumed to be a function of only the weights $w_{i}$. If the error is not acceptable, the weights are updated through various methods. One approach is the gradient descent method, where the weight updates are computed using the derivatives of the error function with respect to the weights:

$$
w_{i}^{(j+1)}=w_{i}^{(j)}-\left.\eta \frac{\partial E}{\partial w_{i}}\right|^{(j)} .
$$

In (6.2) $\eta$ is the learning rate which is used to control the magnitudes of the corrections applied to $w_{i}$. The subscript $j$ indicates the $j$ th iteration. If the value of $\eta$ is too large, the model is prone to convergence issues and at the same time if the value is extremely small the computational time and cost increases. The updated weights are again used for predictions and calculating the error in predictions. The process is repeated until the error is less than a pre-selected value or a maximum number of iterations has been reached. Although (6.2) captures the essence of weight updates, in a typical ANN with multiple hidden layers, the gradient calculation is quite complicated and involved. Hence, in this work, an Adaptive moment estimation (Adam) training algorithm was used for updating the weights. Adam is an algorithm for first-order gradient-based optimization of stochastic objective functions, based on adaptive estimates of lower-order moments. Adam computes individual adaptive learning rates for different parameters from the estimates of first and second moments of the gradients. The algorithm is straight forward to implement and has little memory requirements. For further details on this training algorithm, the reader is referred to [18].

7. ANN modeling and analysis. Python programming language was used to build the ANN model. A high-level neural network API (Application programming interface) Keras was used as the main library. Additional libraries like Pandas, NumPy, and Scikit-learn were used for data preparation and analysis. In this work, both SNNs and DNNs were built, where the desired outputs, specific cutting force $K_{s}$ and maximum tool temperature (MTT) were predicted individually, i.e., the output layer contains only one neuron; either $K_{s}$ or MTT. The input layer has has three input parameters; rake angle $\alpha$, uncut chip thickness $f$, and cutting speed $V_{c}$.

The data sets for training and testing were obtained from FE simulations. Table 7.1 shows the 196 data sets obtained from FEA simulations. The data sets (inputs and outputs) were normalized to the range $[0,1]$ using

$$
V_{\mathrm{N}}=\frac{V-V_{\min }}{V_{\max }-V_{\min }}
$$

Here, $V$ denotes the actual values (which are to be normalized) $V_{\min }, V_{\max }$ denote the minimum and maximum values. Normalizing the data sets is essential to ensure the data sets to be present in a logical correlation. If they are not normalized, the network could possibly consider the data set with higher arithmetic value to be more significant than others. This may affect the generalization ability of the network and can also lead to over fitting [25]. 
The normalized data was split into training and testing sets using an 80:20 ratio. A further $10 \%$ of validation split was performed on the training data set. This was determined to be the most reasonable split after trying different proportions. Thus, the training set consisted of 140 data points, while the validation and test sets consisted of 16 and 40, respectively. During the prediction of specific cutting forces, the maximum tool temperature column from Table 7.1 was excluded, and during the prediction of maximum tool temperature, the cutting force data was excluded.

TABLE 7.1

Data obtained from finite element simulations.

\begin{tabular}{|c|c|c|c|c|c|}
\hline \multirow[b]{2}{*}{ S.No. } & \multicolumn{3}{|c|}{ Input parameters } & \multicolumn{2}{|c|}{ Outputs } \\
\hline & $\begin{array}{l}\text { Rake angle } \\
\left(\operatorname{deg}^{\circ}\right)\end{array}$ & $\begin{array}{l}\text { Uncut chip thickness } \\
\qquad(\mathrm{mm})\end{array}$ & $\begin{array}{l}\text { Cutting speed } \\
(\mathrm{m} / \mathrm{min})\end{array}$ & $\begin{array}{l}\text { Specific cutting force } \\
\qquad\left(\mathrm{N} / \mathrm{mm}^{2}\right)\end{array}$ & $\begin{array}{l}\text { Maximum tool temperature } \\
(\mathrm{K})\end{array}$ \\
\hline 1 & -3 & 0.1 & 100 & 874 & 164 \\
\hline 2 & -3 & 0.1 & 200 & 895 & 184 \\
\hline 3 & -3 & 0.1 & 400 & 945 & 208 \\
\hline . & . & . & . & . & . \\
\hline . & . & . & . & . & . \\
\hline 48 & $\dot{0}$ & 0.3 & 1000 & 667 & 246 \\
\hline 49 & 0 & 0.3 & 1200 & 678 & 249 \\
\hline 50 & 0 & 0.4 & 100 & 681 & 188 \\
\hline . & . & . & · & . & . \\
\hline 84 & 5 & 0.4 & 1200 & 599 & 259 \\
\hline 85 & 8 & 0.1 & 100 & 758 & 150 \\
\hline . & . & . & . & . & . \\
\hline . & . & . & . & . & . \\
\hline 140 & 15 & 0.4 & 1200 & 506 & 229 \\
\hline 141 & 17.5 & 0.1 & 100 & 678 & 144 \\
\hline . & . & . & . & . & . \\
\hline . & . & . & . & . & . \\
\hline . & . & . & . & . & . \\
\hline 195 & 20 & 0.4 & 1000 & 453 & 226 \\
\hline 196 & 20 & 0.4 & 1200 & 495 & 232 \\
\hline
\end{tabular}

Figure 7.1 shows the SNN built in this work for predicting $K_{s}$. We also used the same architecture to predict MTT. During the SNN training, the number of neurons in the hidden layer was varied from five to twenty five and five activation functions (ReLU, ELU, tanh, sigmoid and linear) were employed resulting in $105 \mathrm{SSN}$ architectures for each $K_{s}$ and MTT.

Figure 7.2 shows the DNN built to predict $K_{s}$. We used the same network architecture to predict MTT. In both the cases neurons in the first two hidden layers were varied from five to fifteen, whereas the neurons in the third hidden layer were varied from zero to three. The same five activation functions, mentioned above, were used during the training. A total of 800 DNN architectures were built for predicting each $K_{s}$ and MTT. The number of epochs and batch size were determined to be 150 and 20, respectively, by a trial and error approach. Epochs define how many times the model will be trained through the entire training data set. Batch size determines the number of training samples sent together to the network. For instance, having 1000 data records, setting 10 epochs and batch size of 20 means the network will iterate the training data 10 times. In each iteration, 50 batches are sent to the network and in each batch, the model is trained on 20 data records simultaneously. 


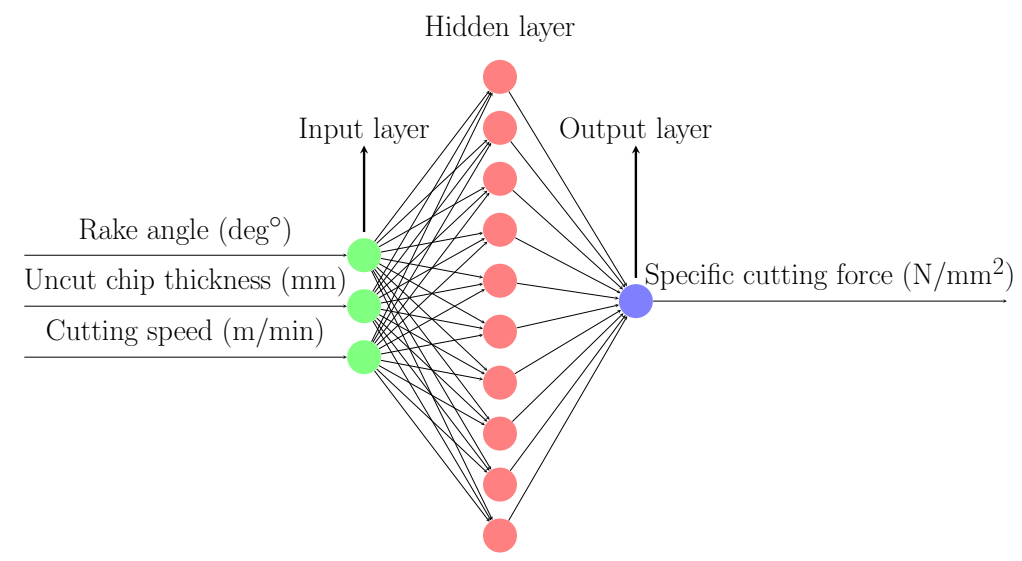

FIG. 7.1. SNN for predicting $K_{s}$.

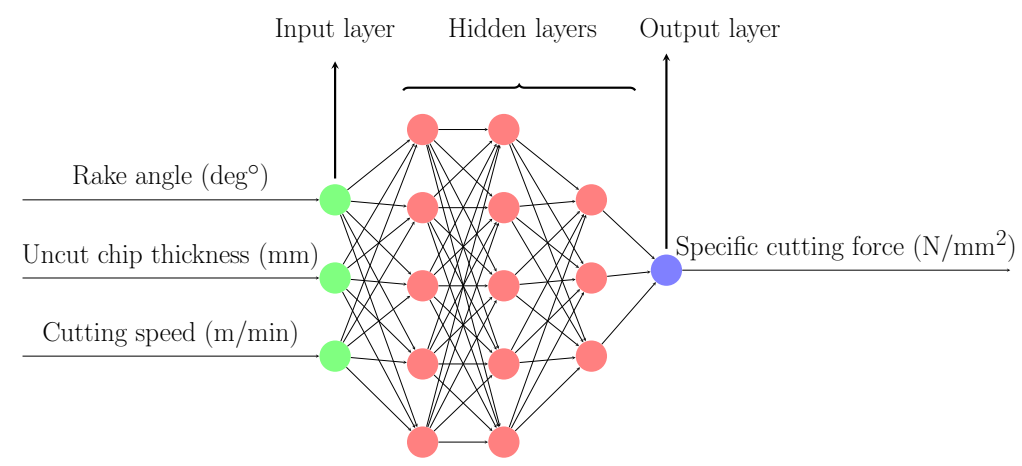

FIG. 7.2. DNN for predicting $K_{s}$.

One important point to be noted is that, linear activation function was used as the default between the output layer and the hidden layer preceding it for all the network architectures. As soon as the training process was completed, the test data sets were fed to the trained neural networks to determine the architecture that exhibited the least error in prediction. For this purpose, statistical evaluations were performed using the mean squared error (MSE)

$$
\mathrm{MSE}=\frac{1}{n} \sum_{i=1}^{n}\left(y_{i}-y_{i}^{p}\right)^{2}
$$

and coefficient of determination $\mathrm{R}^{2}$ using

$$
\mathrm{R}^{2}=1-\frac{\sum_{i=1}^{n}\left(y_{i}-y_{i}^{p}\right)^{2}}{\sum_{i=1}^{n}\left(y_{i}-\bar{y}\right)^{2}} .
$$

In (7.2) and (7.3), $y_{i}$ represents the actual output, $y_{i}^{p}$ represents the ANN predicted output, and $\bar{y}$ represents the mean of the actual outputs. The network architecture with the highest $\mathbf{R}^{2}$ and least MSE on the test data set is concluded to be the suitable network [36]. 
8. Predictions from ANN models. In this section, the results from the two ANN models for the prediction of maximum tool temperature and specific cutting force are presented.

8.1. Prediction of maximum tool temperature. Among the 905 ANN models ( $\mathrm{SNN}+$ DNN) that were built, the model with the network architecture 3-15-14-3-1 with the activation function ReLU has the highest $\mathrm{R}^{2}(0.9605)$ and least MSE $(0.00227)$ on the test data. Figure 8.1 shows the predictions made by this network. It is observed that, the predictions are in close agreement with actual outputs. After examining the predictions, it can be stated that the network architecture 3-15-14-3-1 is the suitable network for predicting the maximum temperature on the cutting tool.

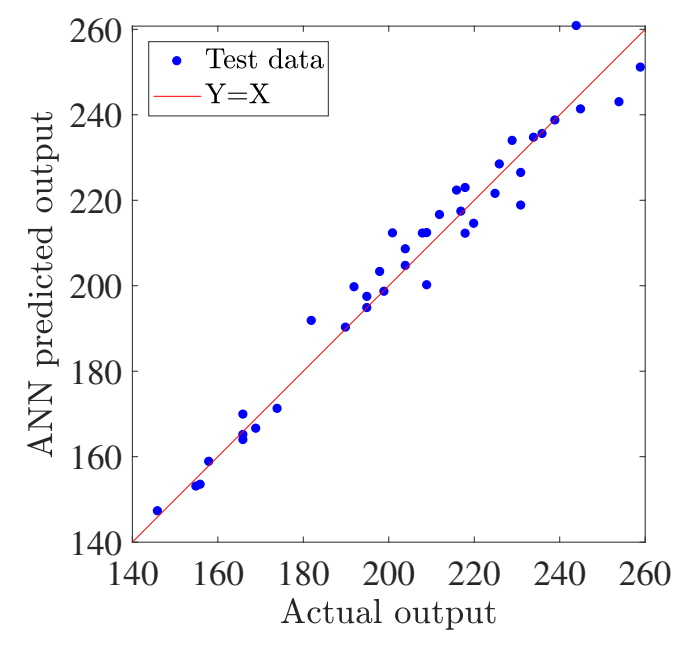

FIG. 8.1. Relation between actual values and ANN predicted values for maximum tool temperature.

TABLE 8.1

Top 10 DNNs and top SNN for predicting maximum tool temperature.

\begin{tabular}{ccccccccc}
\hline & & & & \multicolumn{2}{c}{ Training } & \multicolumn{2}{c}{ Testing } \\
\hline S No & Activation function & Network type & Network architecture & Hidden layers & MSE & $\mathrm{R}^{2}$ & MSE & $\mathrm{R}^{2}$ \\
\hline 1 & ReLU & DNN & $3-15-14-3-1$ & 3 & 0.00113 & 0.9747 & 0.00227 & 0.9605 \\
2 & ReLU & DNN & $3-14-13-3-1$ & 3 & 0.00113 & 0.9748 & 0.00228 & 0.9603 \\
3 & ReLU & DNN & $3-12-13-3-1$ & 3 & 0.00124 & 0.9721 & 0.00235 & 0.9593 \\
4 & ReLU & DNN & $3-11-12-3-1$ & 3 & 0.00144 & 0.9677 & 0.00237 & 0.9588 \\
5 & ReLU & DNN & $3-11-10-3-1$ & 3 & 0.00151 & 0.9662 & 0.00238 & 0.9587 \\
6 & ReLU & DNN & $3-13-13-3-1$ & 3 & 0.00147 & 0.9671 & 0.00248 & 0.9570 \\
7 & ReLU & DNN & $3-13-12-0-1$ & 2 & 0.00140 & 0.9687 & 0.00249 & 0.9568 \\
8 & ReLU & DNN & $3-15-15-0-1$ & 2 & 0.00177 & 0.9603 & 0.00250 & 0.9566 \\
9 & ReLU & DNN & $3-14-14-3-1$ & 3 & 0.00146 & 0.9674 & 0.00256 & 0.9555 \\
10 & ReLU & DNN & $3-11-12-0-1$ & 2 & 0.00166 & 0.9628 & 0.00265 & 0.9540 \\
& ReLU & SNN & $3-23-0-0-1$ & 1 & 0.00187 & 0.9582 & 0.00301 & 0.9477
\end{tabular}

Table 8.1 presents the performance of the top 10 deep neural network architectures and top shallow network arranged in increasing order of MSE (or decreasing order of $\mathrm{R}^{2}$ ) with respect to the test data set. It is interesting to note that neural networks with ReLU as the activation function have performed well compared to other activation functions. 
8.2. Prediction of specific cutting force. Among the 905 ANN models (SNN+DNN), the neural network model with the architecture 3-9-10-0-1 (0 indicates that there are no neurons in the third hidden layer.) with ReLU as the activation function has the highest $\mathrm{R}^{2}(0.9419)$ and least MSE (0.0022) with respect to the test data. After examining the plot (Figure 8.2) which shows the predictions made by this neural network, it can be stated that the network architecture 3-9-10-0-1 predicts outputs closest to the actual outputs.

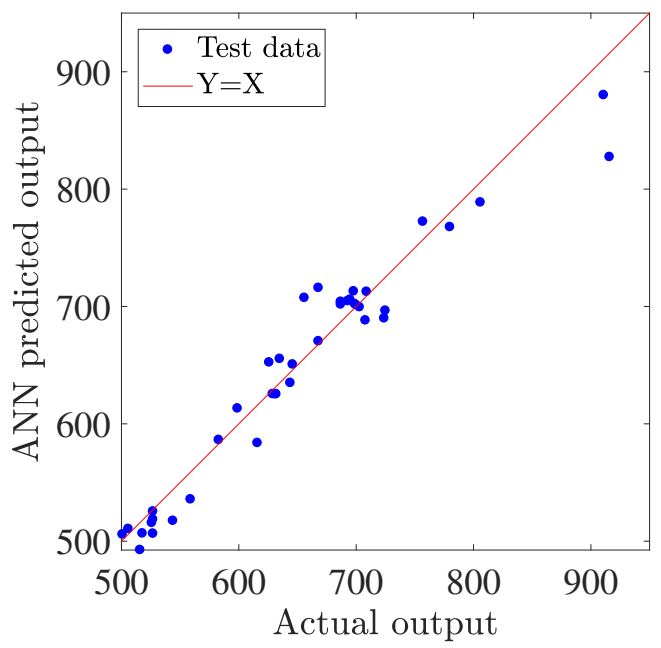

FIG. 8.2. Relation between actual values and ANN predicted values for specific cutting force.

TABLE 8.2

Top 10 DNNs and top SNN for predicting specific cutting force.

\begin{tabular}{ccccccccc}
\hline & & & & \multicolumn{2}{c}{ Training } & \multicolumn{2}{c}{ Testing } \\
\hline S. No & Activation function & Network type & Network architecture & Hidden layers & MSE & $\mathrm{R}^{2}$ & MSE & $\mathrm{R}^{2}$ \\
\hline 1 & ReLU & DNN & $3-9-10-0-1$ & 2 & 0.00294 & 0.9394 & 0.00220 & 0.9419 \\
2 & ReLU & DNN & $3-8-9-0-1$ & 2 & 0.00261 & 0.9463 & 0.00230 & 0.9395 \\
3 & ELU & DNN & $3-13-13-3-1$ & 3 & 0.00290 & 0.9403 & 0.00243 & 0.9359 \\
4 & ReLU & DNN & $3-14-15-3-1$ & 3 & 0.00247 & 0.9492 & 0.00246 & 0.9352 \\
5 & ReLU & DNN & $3-15-15-0-1$ & 2 & 0.00242 & 0.9502 & 0.00248 & 0.9348 \\
6 & ReLU & DNN & $3-13-13-0-1$ & 2 & 0.00282 & 0.9420 & 0.00248 & 0.9347 \\
7 & tanh & DNN & $3-15-14-3-1$ & 3 & 0.00310 & 0.9361 & 0.00253 & 0.9335 \\
8 & tanh & DNN & $3-13-12-3-1$ & 3 & 0.00304 & 0.9375 & 0.00255 & 0.9329 \\
9 & ReLU & DNN & $3-5-5-0-1$ & 2 & 0.00322 & 0.9338 & 0.00258 & 0.9322 \\
10 & ELU & DNN & $3-6-5-0-1$ & 2 & 0.00310 & 0.9362 & 0.00259 & 0.9320 \\
& ReLU & SNN & $3-18-0-0-1$ & 1 & 0.00337 & 0.9305 & 0.00291 & 0.9235
\end{tabular}

Table 8.2 presents the performance of the top 10 deep neural network architectures and top shallow network arranged in increasing order of MSE (or decreasing order of $\mathrm{R}^{2}$ ) with respect to the test data set. It is observed that the neural network with ReLU as the activation function has the best performance, which is similar to the results of maximum tool temperature model.

8.3. Experimental verification. The network architecture 3-9-10-0-1 which was selected for specific cutting force prediction, was further evaluated with the available experimen- 
tal data. That is, the experimental data sets available in the literature $[4,7,19,23]$ were given as the inputs to this neural network and the corresponding outputs $\left(K_{s}\right)$ were predicted and the difference was calculated.

Figure 8.3 shows the actual experimental outputs and the outputs predicted by this neural network. With the exception of a couple of outliers, the predicted values are clearly in good agreement with the experimental values. The corresponding difference in prediction is shown in Figure 8.4. The negative difference for certain data sets indicate that the neural network has over-predicted the experimental output.

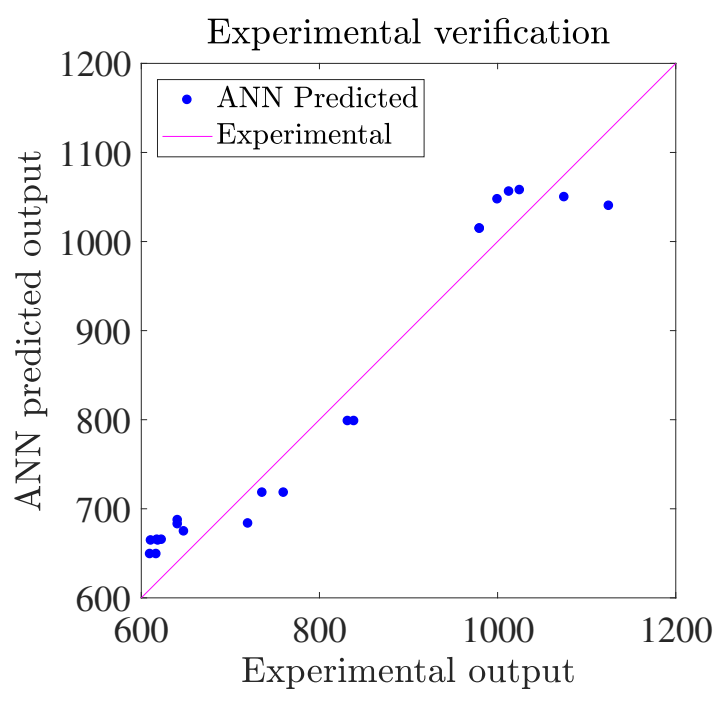

FIG. 8.3. ANN predicted outputs and actual experimental outputs.

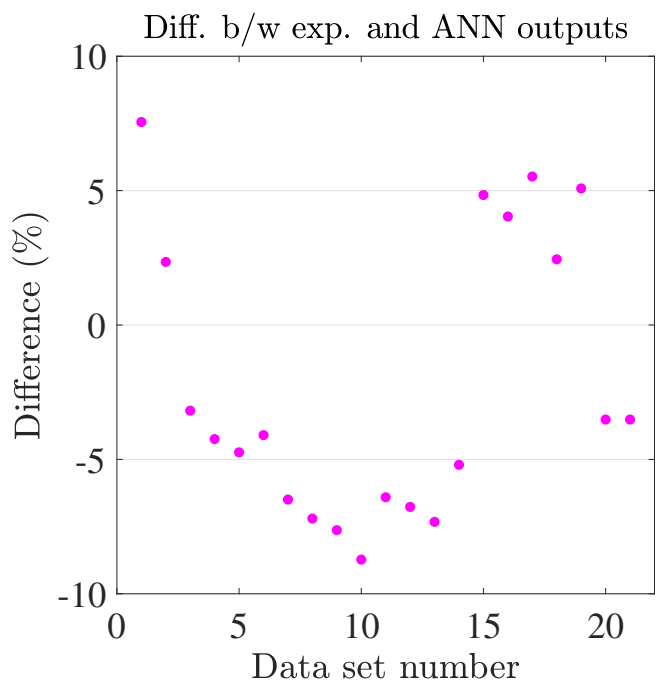

FIG. 8.4. Difference (\%) between experimental output and ANN predicted output. 
9. Sensitivity analysis. Sensitivity studies are extremely important for network designers to predict the effect of input perturbations on the network's output [41]. Sensitivity analysis results tell how likely the outputs based upon the selected model will change on giving new information. The sensitivity of each input is represented by a numerical value, called the sensitivity index.

Sensitivity analysis is carried out by using the open source library SALib [12] available for Python language. This library is capable of generating the model inputs and computing the sensitivity indices from the model outputs. We used the Sobol method [31, 32, 34] available in SALib Package for the purpose of this analysis.

Sobol's method analyzes the portion of variance in the output of the network that is explained by each input variable or each subset of the input variables. Sensitivity indices are available in several forms. We focus on the first-order indices measuring only the effect of a single input variable [12]. First, we provide a brief sketch of the method.

Let $X=\left(x_{1}, \ldots, x_{n}\right)$ denote the input variables of the network and without loss of generality, assume $x_{i} \in[0,1]$. Furthermore, let $Y=\phi(X)$ denote the network output. We may write $Y$ as the sum of simpler orthogonal functions:

$$
Y=\phi(X)=\phi_{0}+\sum_{i=1}^{n} \phi_{i}\left(x_{i}\right)+\sum_{i<j}^{n} \phi_{i j}\left(x_{i}, x_{j}\right)+\cdots+\phi_{12 \ldots n}\left(x_{1}, x_{2}, \ldots, x_{n}\right)
$$

In (9.1) $\phi_{0}$ is a constant, $\phi_{i}$ s are single variable functions, and all the other terms are multivariable functions. A sufficient condition for the existence of such orthogonal terms is that the condition

$$
\begin{array}{r}
\int_{0}^{1} \phi_{j_{r}, \ldots, j_{t}}\left(x_{j_{r}}, \ldots, x_{j_{t}}\right) d x_{i}=0 \quad \text { for } \quad i=j_{r}, \ldots, j_{t} \\
\text { where } \quad 1 \leq j_{r}<\cdots<j_{t} \leq n
\end{array}
$$

holds.

Assuming all of the functions in (9.1) are square integrable on $I=[0,1]$, we can define their variances. For the output $Y$ we have:

$$
\begin{aligned}
V=\operatorname{Var}(Y) & =\int_{I^{n}} \phi^{2}(X) d X-\phi_{0}^{2} \\
& =\int_{I} \cdots \int_{I} \phi^{2}\left(x_{1}, x_{2}, \ldots, x_{n}\right) d x_{1} \cdots d x_{n}-\phi_{0}^{2} .
\end{aligned}
$$

For the right hand terms (except the constant $\phi_{0}$ ) we may define the variances as in (9.4):

$$
\begin{array}{r}
V_{j_{r}, \ldots, j_{t}}=\int \phi_{j_{r}, \ldots, j_{t}}^{2}\left(x_{j_{r}}, \ldots, x_{j_{t}}\right) d x_{j_{r}} \cdots d x_{j_{t}} \\
\text { where } \quad 1 \leq j_{r}<\cdots<j_{t} \leq n .
\end{array}
$$

Of course, the orthogonality condition guarantees that the variance of sum equals the sum of variances, as in (9.5),

$$
V=\operatorname{Var}(Y)=\sum_{i=1}^{n} V_{i}+\sum_{i<j}^{n} V_{i j}+\cdots+V_{1 \ldots n} .
$$

Now, we can define the sensitivity indices as:

$$
\psi_{j_{r} \ldots j_{t}}=\frac{V_{j_{r}, \ldots, j_{t}}}{V} .
$$




\section{ETNA}

Kent State University and

Johann Radon Institute (RICAM)

Comparing with (9.5), we can immediately observe that the sum of all sensitivity indices is equal to one:

$$
\sum_{t=1}^{n} \sum_{j_{r}<\cdots<j_{t}} \psi_{j_{r} \ldots j_{t}}=1
$$

In (9.6), $\psi_{j_{r} \ldots j_{t}}$ measures the joint effect of $x_{r} \ldots x_{t}$ on the output $Y$. As all $\left(2^{n}-1\right)$ non-empty subsets of the input variables are presented in (9.6), it is not easy to interpret the entire results. But we may focus only on the exclusive direct sensitivity of $Y$ to each of the input variables alone using

$$
\psi_{i}=\frac{V_{i}}{V} .
$$

Here, in (9.8), $\psi_{i}$ 's are referred to as the first order indices. Each $\psi_{i}$ measures the portion of variance in $Y$ exclusively explained by $x_{i}$. These indices are all we utilize for the purpose of this study. Such indices are usually calculated easily via Monte Carlo simulations [31, 34].

We refer the interested reader to [34] for the details of the Sobol's method. In our study, we used three model inputs (rake angle $(\alpha)$, uncut chip thickness $(f)$ and cutting speed $\left(V_{c}\right)$ ). The results of sensitivity analysis on the selected neural network architectures, for specific cutting forces $\left(K_{s}\right)$ and maximum tool temperatures (MTT), are shown in Table 9.1. For specific cutting forces both the rake angle and uncut chip thickness have more impact on the output compared to the cutting speed, whereas for maximum tool temperatures cutting, speed seems to have more effect on the output compared to both the rake angle and uncut chip thickness.

TABLE 9.1

Sensitivity indices for SCF and MTT.

First-order indices

\begin{tabular}{lcc}
\hline Parameter & $K_{s}(3-9-10-0-1)$ & MTT (3-15-14-3-1) \\
\hline Rake angle, $(\alpha)$ & 0.5319 & 0.2206 \\
Uncut chip thickness, $(f)$ & 0.4452 & 0.1316 \\
Cutting speed, $\left(V_{c}\right)$ & 0.0009 & 0.6160
\end{tabular}

The sensitivity analysis results were verified with the results obtained from finite element simulations. Figure 9.1 shows variation in specific cutting forces for rake angles $8^{\circ}$ and $-3^{\circ}$ during the cutting speeds $200 \mathrm{~m} / \mathrm{min}$ and $600 \mathrm{~m} / \mathrm{min}$ for various uncut chip thickness values. It is inferred that specific cutting forces are changing rapidly with the change in uncut chip thickness and rake angle, but they remain almost the same for different cutting speeds. The same results can be concluded from Table 9.1. The sensitivity of cutting speed on specific cutting force is 0.0009 showing that small changes of cutting speed do not have a noticeable impact on specific cutting force. Also, the sensitivity of specific cutting force to rake angle and uncut chip thickness is much higher than cutting speed.

On the other hand, Figure 9.2 shows the variation in maximum tool temperatures; it can be seen that the temperature is changing rapidly with the increase in cutting speeds but does not show much variation with rake angles $\alpha$ and uncut chip thickness $f$. This is a reasonable result since according to Table 9.1, the impact of cutting speed on tool temperature is higher than the rake angle and uncut ship thickness. Hence, we can conclude that the results obtained from sensitivity analysis are in good agreement with the finite element simulations. 


\section{ETNA}

Kent State University and

Johann Radon Institute (RICAM)

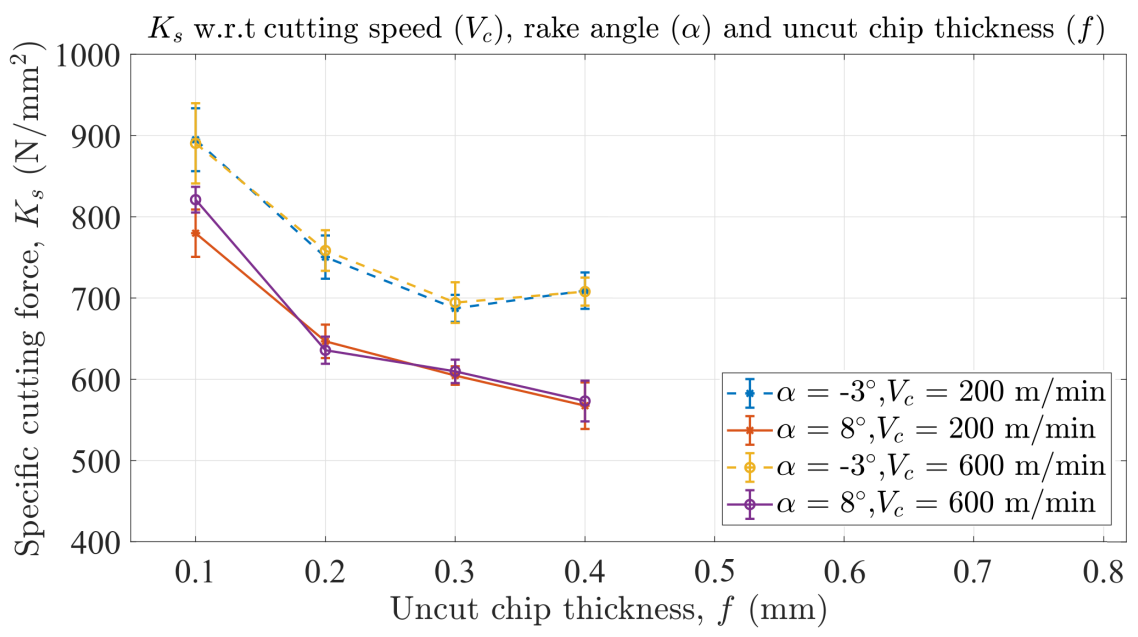

FIG. 9.1. Specific cutting forces obtained from FEA simulations.

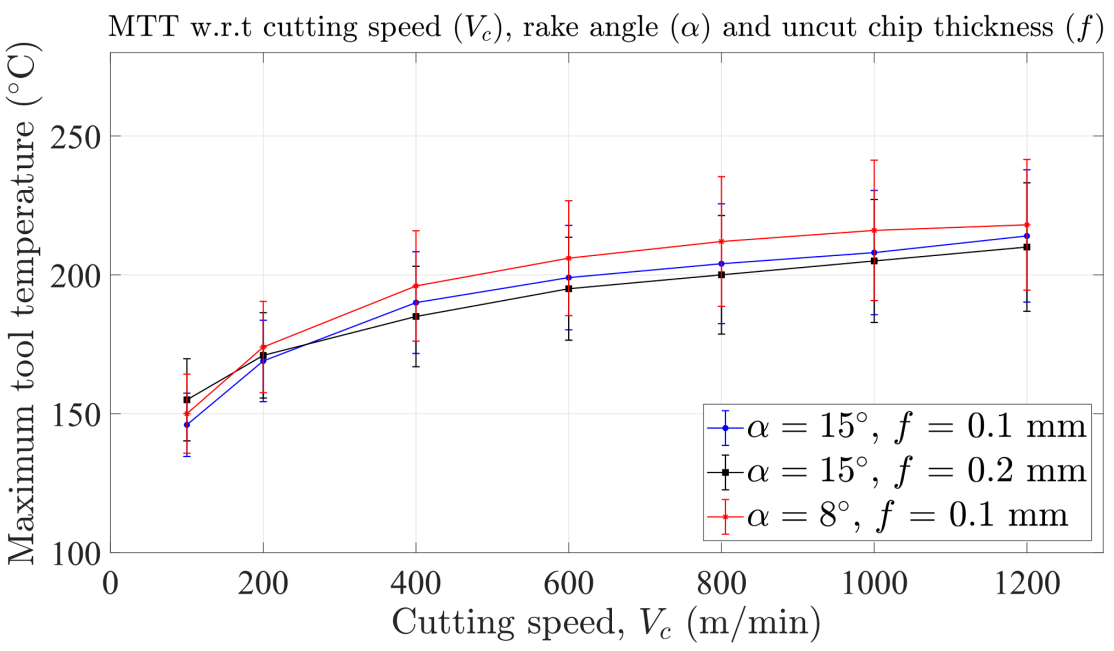

FIG. 9.2. Maximum tool temperatures obtained from FEA simulations.

10. Conclusions. The paper presented a comprehensive analysis of the application of FEM and ANN to predict specific cutting forces and maximum tool temperatures, including a detailed description of modeling orthogonal machining. A total of 196 simulations were performed for various rake angles, uncut chip thickness, and cutting speeds for generating data. In this study, 905 neural network models were built for each specific cutting force and maximum tool temperature prediction. The suitable neural network architecture for predicting specific cutting forces is found to be 3-9-10-0-1, with ReLU as the activation function, whereas for predicting maximum tool temperatures the neural network architecture 3-15-14-3-1, with ReLU as the activation function, was found to be suitable. Sensitivity analysis was performed to check the sensitivity of the output with input perturbations, and results revealed that, specific cutting forces are sensitive to both rake angle and uncut chip thickness. On the other hand, maximum tool temperatures were found to be sensitive to cutting speeds. 
The work reveals that the hybrid approach of combining FEM and machine learning to predict specific cutting forces and maximum tool temperatures is effective. The coefficient of determination $\mathrm{R}^{2}$ can be improved by adding more number of data sets during the ANN modeling process.

The proposed approach can be extended for other work materials and manufacturing applications. Additional parameters like stresses, strains, and tool-tip temperatures can be predicted. More advanced training algorithms, such as Nadam and Adamax, can be used along with the application of other activation functions, including leaky ReLU, PReLU, and Thresholded ReLU.

11. Acknowledgements. The Center for Self-Aware Manufacturing and Metrology, and the research described in this paper, are supported under a multi-year grant from the University of North Carolina's Research Opportunities Initiative. The first author wishes to thank Nishant Ojal for helping making the neural network figures in this paper.

\section{REFERENCES}

[1] Abaqus 2017 documentation. http://130.149.89.49:2080/v2016/index.html.

[2] A. H. ADIBI-SEDEH, V. MADHAVAN, AND B. BAHR, Extension of Oxley's analysis of machining to use different material models, J. Manuf. Sci. Eng., 125 (2003), pp. 656-666.

[3] A. Al-Ahmari, Predictive machinability models for a selected hard material in turning operations, J. Mater. Process. Technol., 190 (2007), pp. 305-311.

[4] M. Asad, F. Girardin, T. Mabrouki, And J.-F. Rigal, Dry cutting study of an aluminium alloy (A2024-T351): a numerical and experimental approach, Int. J. Mater. Form., 1 (2008), pp. 499-502.

[5] P. Asokan, R. R. Kumar, R. Jeyapaul, AND M. SANThI, Development of multi-objective optimization models for electrochemical machining process, Int. J. Adv. Manuf. Technol., 39 (2008), pp. 55-63.

[6] V. P. Astakhov and S. ShVets, The assessment of plastic deformation in metal cutting, J. Mater. Process. Technol., 146 (2004), pp. 193-202.

[7] S. Atlati, B. HadDaG, M. NOUARI, AND M. Zenasni, Analysis of a new segmentation intensity ratio "SIR" to characterize the chip segmentation process in machining ductile metals, Int. J. Mach. Tools Manuf., 51 (2011), pp. 687-700.

[8] H. Cherukuri, E. Perez-Bernabeu, M. Selles, And T. L. Schmitz, A neural network approach for chatter prediction in turning, Procedia Manuf., 34 (2019), pp. 885-892.

[9] H. Cherukuri, E. Perez-Bernabeu, M. A. Selles, And T. Schmitz, Machining chatter prediction using a data learning model, J. Manuf. Mater. Process., 3 (2019).

[10] M. Correa, C. Bielza, And J. Pamies-Teixeira, Comparison of Bayesian networks and artificial neural networks for quality detection in a machining process, Expert Syst. Appl., 36 (2009), pp. 7270-7279.

[11] S. S. HAY KIN, Neural Networks and Learning Machines, 3rd. ed., Pearson, Upper Saddle River, 2009.

[12] J. HERMAN AND W. UsHer, SALib: An open-source Python library for sensitivity analysis, J. Open Source Softw., 2 (2017), doi:10.21105/joss.00097.

[13] Y. HUANG AND S. LIANG, Cutting forces modeling considering the effect of tool thermal property-application to CBN hard turning, Int. J. Mach. Tools Manuf., 43 (2003), pp. 307-315.

[14] G. JOHNSON AND W. COOK, A constitutive model and data for metals subjected to large strains, strain rates, and high pressures, in Proceedings of the 7th International Symposium On Ballistics, The Hague, 1983, pp. 541-548.

[15] G. R. JOHNSON AND W. H. COOK, Fracture characteristics of three metals subjected to various strains, strain rates, temperatures and pressures, Eng. Fract. Mech., 21 (1985), pp. 31-48.

[16] F. KARA, K. ASLANTAS, AND A. ÇIÇEK, ANN and multiple regression method-based modelling of cutting forces in orthogonal machining of AISI 316L stainless steel, Neural Comput. Appl., 26 (2015), pp. 237250.

[17] D. E. Kim AND M. Gofman, Comparison of shallow and deep neural networks for network intrusion detection, in 2018 IEEE 8th Annual Computing and Communication Workshop and Conference (CCWC), IEEE Conference Proceedings, Los Alamitos, 2018, pp. 204-208.

[18] D. P. KIngma AND J. BA, Adam: A method for stochastic optimization, arXiv Preprint, arXiv:1412.6980, 2014. https://arxiv.org/abs/1412.6980.

[19] S. Kobayashi, R. Herzog, D. EgGleston, and E. Thomsen, A critical comparison of metal-cutting theories with new experimental data, J. Eng. Ind., 82 (1960), pp. 333-347.

[20] S. Kouadri, K. Necib, S. ATlati, B. HAdDAG, AND M. NouARI, Quantification of the chip segmentation 
in metal machining: Application to machining the aeronautical aluminium alloy AA2024-T351 with cemented carbide tools WC-Co, Int. J. Mach. Tools Manuf., 64 (2013), pp. 102-113.

[21] S. LIANG AND R. SRIKANT, Why deep neural networks for function approximation?, arXiv Preprint, arXiv:1610.04161, 2016. https://arxiv.org/abs/1610.04161.

[22] T. Mabrouki, F. Girardin, M. Asad, And J.-F. Rigal, Numerical and experimental study of dry cutting for an aeronautic aluminium alloy (A2024-T351), Int. J. Mach. Tools Manuf., 48 (2008), pp. 1187-1197.

[23] M. MADAJ AND M. PÍŠKA, On the SPH orthogonal cutting simulation of A2024-T351 alloy, Procedia CIRP, 8 (2013), pp. 152-157.

[24] A. P. Markopoulos, Finite Element Method in Machining Processes, Springer, London, 2012.

[25] A. P. Markopoulos, D. E. Manolakos, And N. M. VAXEVAnidis, Artificial neural network models for the prediction of surface roughness in electrical discharge machining, J. Intell. Manuf., 19 (2008), pp. 283-292.

[26] İ. OVALI AND A. MAVI, A study on cutting forces of austempered gray iron using artificial neural networks, Eng. Sci. Technol. an Int., 16 (2013) pp. 1-10.

[27] T. ÖZEL AND E. ZEREN, A methodology to determine work material flow stress and tool-chip interfacial friction properties by using analysis of machining, J. Manuf. Sci. Eng., 128 (2006), pp. 119-129.

[28] J. PATEL AND H. P. CHERUKURI, Chip morphology studies using separate fracture toughness values for chip separation and serration in orthogonal machining simulations, in ASME 2018 13th International Manufacturing Science and Engineering Conference, American Society of Mechanical Engineers, New York, 2018, V002T04A031.

[29] J. P. PATEL, Finite Element Studies of Orthogonal Machining of Aluminum Alloy A2024-T351, PhD. Thesis, The University of North Carolina, Charlotte, 2018.

[30] F. J. Pontes, J. R. Ferreira, M. B. Silva, A. P. Paiva, and P. P. Balestrassi, Artificial neural networks for machining processes surface roughness modeling, Int. J. Adv. Manuf. Technol., 49 (2010), pp. 879-902.

[31] A. SAltelli, Making best use of model evaluations to compute sensitivity indices, Comput. Phys. Commun., 145 (2002), pp. 280-297.

[32] A. Saltelli, P. Annoni, I. Azzini, F. Campolongo, M. Ratto, and S. Tarantola, Variance based sensitivity analysis of model output. design and estimator for the total sensitivity index, Comput. Phys. Commun., 181 (2010), pp. 259-270.

[33] A. SCHINDLER, T. LIDY, AND A. RAUBER, Comparing shallow versus deep neural network architectures for automatic music genre classification, in Proceedings of the 9th Forum Media Technology (FMT2016), W. Aigner, G. Schmiedl, K. Blumenstein, eds., Lulu.com, Morrisville, 2016, pp. 17-21.

[34] I. M. SobOL, Global sensitivity indices for nonlinear mathematical models and their Monte Carlo estimates, Math. Comput. Simulation, 55 (2001), pp. 271-280.

[35] S. TASDEMIR, Artificial neural network based on predictive model and analysis for main cutting force in turning, Energy Education Science and Technology Part A-Energy Science and Research, 29 (2012), pp. 1471-1480.

[36] S. TASDEMIR, Artificial neural network model for prediction of tool tip temperature and analysis, Int. J. Intell. Syst. Appl. Eng, 6 (2018), pp. 92-96.

[37] X. TENG AND T. WIERZBICKI, Evaluation of six fracture models in high velocity perforation, Eng. Fract. Mech., 73 (2006), pp. 1653-1678.

[38] A. E. TUMER AND S. EDEBALI, An artificial neural network model for wastewater treatment plant of Konya, Int. J. Intell. Syst. Appl. Eng., 3 (2015), pp. 131-135.

[39] P. WAllace AND G. BoOThROYD, Tool forces and tool-chip friction in orthogonal machining, J. Mech. Eng. Sci., 6 (1964), pp. 74-87.

[40] X. YANG AND C. R. LIU, A new stress-based model of friction behavior in machining and its significant impact on residual stresses computed by finite element method, Int. J. Mech. Sci., 44 (2002), pp. 703-723.

[41] X. ZENG AND D. S. YEUNG, Sensitivity analysis of multilayer perceptron to input and weight perturbations, IEEE Trans. Neural Netw., 12 (2001), pp. 1358-1366.

[42] N. ZOREV, Inter-relationship between shear processes occurring along tool face and shear plane in metal cutting, Int. Res. Prod. Eng., 49 (1963), pp. 143-152. 